\title{
SLANT SUBMANIFOLDS IN SASAKIAN MANIFOLDS
}

\author{
J. L. CABRERIZO, A. CARRIAZO, L. M. FERNÁNDEZ \\ and M. FERNÁNDEZ ${ }^{1}$ \\ Departamento de Geometría y Topología, Facultad de Matemáticas, Universidad de Sevilla, \\ Apartado de Correos 1160, 41080 - Sevilla, Spain \\ e-mail:acarri@cica.es
}

(Received 5 August, 1998)

\begin{abstract}
In this paper, we show new results on slant submanifolds of an almost contact metric manifold. We study and characterize slant submanifolds of $K$ contact and Sasakian manifolds. We also study the special class of three-dimensional slant submanifolds. We give several examples of slant submanifolds.

1991 Mathematics Subject Classification. 53C15, 53C40.
\end{abstract}

0. Introduction. Slant immersions in complex geometry were defined by B.-Y. Chen as a natural generalization of both holomorphic immersions and totally real immersions [2]. Examples of slant immersions into complex Euclidean spaces $\mathbf{C}^{2}$ and $\mathbf{C}^{4}$ were given by Chen and Tazawa $[\mathbf{2}, \mathbf{4}, \mathbf{5}]$, while slant immersions of Kähler $\mathbf{C}$ spaces into complex projective spaces were given by Maeda, Ohnita and Udagawa [9].

In a recent paper [7], A. Lotta has introduced the notion of slant immersion of a Riemannian manifold into an almost contact metric manifold and he has proved some properties of such immersions. A. Lotta and A. M. Pastore have obtained examples of slant submanifolds in the Sasakian-space-form $\mathbf{R}^{2 m+1}$ as the leaves of a harmonic Riemannian 3-dimensional foliation [8]. Finally, A. Lotta has also studied some properties about the intrinsic geometry of 3-dimensional non-anti-invariant slant submanifolds of $K$-contact manifolds [6].

The purpose of the present paper is to study slant immersions in $K$-contact and Sasakian manifolds. We first review, in Section 1, basic formulas and definitions for almost contact metric manifolds and their submanifolds, which we shall use later. In Section 2, we recall the definition of a slant submanifold of an almost contact metric manifold and we show a first characterization theorem. In Section 3, we give many interesting examples of slant submanifolds in almost contact metric manifolds and in Sasakian manifolds. Then, we characterize slant submanifolds by means of the covariant derivative of the square of the tangent projection $T$ over the submanifold of the almost contact structure of a $K$-contact manifold. Later, we study the first interesting class of slant submanifolds: the three-dimensional slant submanifolds. We show some results concerning the tangent $T$ and the normal $N$ projections. We also use the given examples in order to remark some facts concerning the main theorems of the paper. We study slant submanifolds in $K$-contact manifolds and threedimensional slant submanifolds in Sections 4 and 5 respectively.

\footnotetext{
${ }^{1}$ The authors are partially supported by the PAI project (Junta de Andalucía, Spain 1998).
} 
1. Preliminaries. Let $(\tilde{M}, g)$ be an odd-dimensional Riemannian manifold and denote by $T \widetilde{M}$ the Lie algebra of vector fields in $\widetilde{M}$. Then, $\widetilde{M}$ is said to be an almost contact metric manifold $[\mathbf{1}]$ if there exists on $\widetilde{M}$ a tensor $\phi$ of type $(1,1)$ and a global vector field $\xi$ (structure vector field) such that, if $\eta$ is the dual 1 -form of $\xi$, then

$$
\begin{gathered}
\phi^{2} X=-X+\eta(X) \xi, \quad g(X, \xi)=\eta(X), \\
g(\phi X, \phi Y)=g(X, Y)-\eta(X) \eta(Y),
\end{gathered}
$$

for any $X, Y \in T \tilde{M}$. In this case,

$$
g(\phi X, Y)+g(X, \phi Y)=0
$$

for any $X, Y \in T \tilde{M}$. Let $\Phi$ denote the 2-form in $\tilde{M}$ given by $\Phi(X, Y)=g(X, \phi Y)$ for all $X, Y \in T \tilde{M}$. The 2-form $\Phi$ is called the fundamental 2-form in $\widetilde{M}$ and the manifold is said to be a contact metric manifold if $\Phi=\mathrm{d} \eta$. If $\xi$ is a Killing vector field with respect to $\mathrm{g}$, the contact metric structure is called a $K$-contact structure. It is easy to prove that a contact metric manifold is $K$-contact if and only if $\widetilde{\nabla}_{X} \xi=-\phi X$, for any $X \in T \widetilde{M}$, where $\widetilde{\nabla}$ denotes the Levi-Civita connection of $\widetilde{M}$.

The almost contact structure of $\widetilde{M}$ is said to be normal if $[\phi, \phi]+2 \mathrm{~d} \eta \otimes \xi=0$, where $[\phi, \phi]$ is the Nijenhuis torsion of $\phi$. A Sasakian manifold is a normal contact metric manifold. Every Sasakian manifold is a $K$-contact manifold. It is easy to show that an almost contact metric manifold is a Sasakian manifold if and only if

$$
\left(\widetilde{\nabla}_{X} \phi\right) Y=g(X, Y) \xi-\eta(Y) X
$$

for any $X, Y \in T \tilde{M}$.

Now, let $M$ be a submanifold immersed in $\tilde{M}$. We also denote by $g$ the induced metric on $M$. Let $T M$ be the Lie algebra of vector fields in $M$ and $T^{\perp} M$ the set of all vector fields normal to $M$. Denote by $\nabla$ the Levi-Civita connection of $M$. Then, the Gauss-Weingarten formulas are given by

$$
\widetilde{\nabla}_{X} Y=\nabla_{X} Y+\sigma(X, Y), \quad \widetilde{\nabla}_{X} V=-A_{V} X+D_{X} V
$$

for any $X, Y \in T M$ and any $V \in T^{\perp} M$, where $D$ is the connection in the normal bundle, $\sigma$ is the second fundamental form of $M$ and $A_{V}$ is the Weingarten endomorphism associated with $V$.

The mean curvature vector $H$ is defined by $H=(1 / m)$ trace $\sigma$, where $m$ is the dimension of $M . M$ is said to be minimal if $H$ vanishes identically.

For any $X \in T M$, we write

$$
\phi X=T X+N X
$$

where $T X$ is the tangential component of $\phi X$ and $N X$ is the normal component of $\phi X$. Similarly, for any $V \in T^{\perp} M$, we have

$$
\phi V=t V+n V
$$

where $t V$ (resp. $n V$ ) is the tangential component (resp. normal component) of $\phi V$. 
From (1.2) and (1.4), we get

$$
g(T X, Y)=-g(X, T Y)
$$

for any $X, Y \in T M$.

The submanifold $M$ is said to be invariant if $N$ is identically zero, that is, $\phi X \in T M$, for any $X \in T M$. On the other hand, $M$ is said to be an anti-invariant submanifold if $T$ is identically zero, that is, $\phi X \in T^{\perp} M$, for any $X \in T M$.

From now on, we put $Q=T^{2}$. We define $\nabla Q, \nabla T$ and $\nabla N$ by $\left(\nabla_{X} Q\right) Y=$ $\nabla_{X}(Q Y)-Q\left(\nabla_{X} Y\right),\left(\nabla_{X} T\right) Y=\nabla_{X}(T Y)-T\left(\nabla_{X} Y\right)$ and $\left(\nabla_{X} N\right) Y=D_{X} N Y-N \nabla_{X} Y$, for any $X, Y \in T M$.

It can be proved by a direct calculation that if there exists a function $\lambda$ such that

$$
\left(\nabla_{X} T\right) Y=\lambda(g(X, Y) \xi-\eta(Y) X),
$$

for any $X, Y \in T M$, then

$$
\left(\nabla_{X} Q\right) Y=\lambda(g(X, T Y) \xi-\eta(Y) T X),
$$

for any $X, Y \in T M$.

Suppose now that $M$ is a submanifold of a Sasakian manifold $\tilde{M}$. Then, by applying the formulas of Gauss and Weingarten and using formulas (1.3), (1.4) and (1.5), we may obtain $\left(\nabla_{X} T\right) Y=t \sigma(X, Y)+A_{N Y} X+g(X, Y) \xi-\eta(Y) X$ and $\left(\nabla_{X} N\right) Y=n \sigma(X, Y)-\sigma(X, T Y)$, for any vectors $X, Y$ tangent to $M$. Hence, if $\lambda$ is a function, then, it can be proved that

$$
\left(\nabla_{X} T\right) Y=\lambda(g(X, Y) \xi-\eta(Y) X),
$$

for any $X, Y \in T M$, if and only if

$$
A_{N Y} X=A_{N X} Y+(1-\lambda) \widetilde{R}(X, Y) \xi
$$

for any $X, Y \in T M$. Similarly, we can see that

$$
\left(\nabla_{X} N\right) Y=2 \eta(X) N T Y+\eta(Y) N T X,
$$

for any $X, Y \in T M$ if and only if

$$
A_{V} T Y=-A_{n V} Y+2 g(Y, \operatorname{tn} V) \xi+\eta(Y) \operatorname{tn} V,
$$

for any $Y \in T M$ and any $V \in T M^{\perp}$.

2. Slant submanifolds. In [7], A. Lotta has introduced the following notion of slant immersion in almost contact metric manifolds. A submanifold $M$ is said to be slant if for any $x \in M$ and any $X \in T_{x} M$, linearly independent on $\xi$, the angle between $\phi X$ and $T_{x} M$ is a constant $\theta \in[0, \pi / 2]$, called the slant angle of $M$ in $\widetilde{M}$.

Invariant and anti-invariant immersions are slant immersions with slant angle $\theta=0$ and $\theta=\pi / 2$ respectively. A slant immersion which is not invariant nor anti-invariant is called a proper slant immersion. 
Lotta's definition includes both $\xi \in T M$ and $\xi \in T^{\perp} M$ cases. Nevertheless, he proves the following theorem, which generalizes a well-known result of Yano and Kon (see Proposition 7.3 of [10]):

THeORem 2.1. [7] Let $M$ be a submanifold of a contact metric manifold $\tilde{M}$. If $\xi$ is orthogonal to $M$, then $M$ is anti-invariant.

Given that we are interested in the study of proper slant submanifolds in contact metric manifolds, from now on we suppose that the structure vector field is tangent to $M$. Hence, if we denote by $\mathcal{D}$ the orthogonal distribution to $\xi$ in $T M$, we can consider the orthogonal direct decomposition: $T M=\mathcal{D} \oplus<\xi>$.

We have already noted down that invariant and anti-invariant submanifolds are special classes of slant submanifolds. In the first case, we know that $N \equiv 0$, so that $T=\phi$ and therefore $T^{2}=\phi^{2}=-I+\eta \otimes \xi$. For an anti-invariant submanifold, we have $T^{2}=0$. If $M$ is a proper slant submanifold, with slant angle $\theta$, Lotta has proved in [7] that $T^{2} X=-\cos ^{2} \theta(X-\eta(X) \xi)$, for any $X \in T M$. It is clear that this expression includes invariant and anti-invariant cases, for $\theta=0$ and $\theta=\pi / 2$, respectively. In fact, the following result shows that it characterizes slant immersions.

THeORem 2.2. Let $M$ be a submanifold of an almost contact metric manifold $\widetilde{M}$ such that $\xi \in T M$. Then, $M$ is slant if and only if there exists a constant $\lambda \in[0,1]$ such that:

$$
T^{2}=-\lambda(I-\eta \otimes \xi)
$$

Furthermore, in such case, if $\theta$ is the slant angle of $M$, it satisfies that $\lambda=\cos ^{2} \theta$.

Proof. We only have to prove the sufficient condition. Suppose that there exists a constant $\lambda$ such that $T^{2}=-\lambda(I-\eta \otimes \xi)$. Then, for any $X \in T M-<\xi>$ we have:

$$
\cos \theta(X)=\frac{g(\phi X, T X)}{|\phi X||T X|}=-\frac{g\left(X, T^{2} X\right)}{|\phi X||T X|}=-\lambda \frac{g\left(X, \phi^{2} X\right)}{|\phi X||T X|}=\lambda \frac{|\phi X|}{|T X|}
$$

On the other hand, $\cos \theta(X)=|T X| /|\phi X|$, and so, by using (2.2), we obtain $\cos ^{2} \theta(X)=\lambda$. Hence, $\theta(X)$ is a constant and so $M$ is slant.

COROLlary 2.3. Let $M$ be a slant submanifold of a almost contact metric manifold $\widetilde{M}$, with slant angle $\theta$. Then, for any $X, Y \in T M$, we have:

$$
\begin{aligned}
& g(T X, T Y)=\cos ^{2} \theta(g(X, Y)-\eta(X) \eta(Y)), \\
& g(N X, N Y)=\sin ^{2} \theta(g(X, Y)-\eta(X) \eta(Y)) .
\end{aligned}
$$

Proof. From (1.6) and (2.1), a direct expansion gives (2.3). To prove (2.4), it is enough to take into account (1.1) and (1.4). 
3. Examples. In this section we give some examples of slant submanifolds in almost contact metric manifolds. Our goal is to find interesting examples of proper slant submanifolds in Sasakian manifolds.

An important work in the study of slant submanifolds in the almost complex case is the characterization of slant surfaces. Examples and properties of slant surfaces are given in $[\mathbf{2}, \mathbf{3}, \mathbf{4}, \mathbf{5}]$. However, Lotta has proved that a non-anti-invariant slant submanifold of a contact metric manifold must have an odd dimension and so, it follows that surfaces are not interesting in our research. Then, to obtain examples of slant submanifolds in our case, we must look for submanifolds with dimension greater or equal to 3 . Lotta has already found the following example:

ExAmple 3.1. [7] If $M$ is a slant submanifold in an almost Hermitian manifold $\tilde{M}$, then $M \times \mathbf{R}$ is a slant submanifold in the almost contact metric manifold $\widetilde{M} \times \mathbf{R}$ with the usual product structure.

Thus, each example of $[\mathbf{2}, \mathbf{3}, \mathbf{4}, \mathbf{5}]$, provides an example of slant submanifold in an almost contact metric manifold.

Then, we could think that, if $M$ is a slant submanifold of a Kaehlerian manifold $\tilde{M}, M \times R$ would be a slant submanifold in a certain Sasakian manifold. However, we have the following proposition.

Proposition 3.2. Let $M$ be a submanifold of a contact metric manifold $\tilde{M}$. Then, $M$ is anti-invariant if and only if the distribution $\mathcal{D}$ is integrable.

Proof. It is enough to take into account that, if $\tilde{M}$ is contact metric, then, for any $X, Y \in T M$ :

$$
2 g(X, T Y)=2 \mathrm{~d} \eta(X, Y)=X(\eta(Y))-Y(\eta(X))-\eta([X, Y]) .
$$

In particular, $2 g(X, T Y)=-\eta([X, Y])$ for any $X, Y \in \mathcal{D}$, which implies that $\mathcal{D}$ is integrable if and only if $T \equiv 0$.

Hence, if $M$ is a slant submanifold in an almost Hermitian manifold, $M \times \mathbf{R}$ can not be a proper slant submanifold in a contact metric manifold. Note that $\widetilde{M} \times \mathbf{R}$ is not contact metric and so there is not any contradiction with Example 3.1.

From now on, $\left(\mathbf{R}^{2 m+1}, \phi_{0}, \xi, \eta, g\right)$ will denote the manifold $\mathbf{R}^{2 m+1}$ with its usual Sasakian structure given by

$$
\begin{gathered}
\eta=1 / 2\left(d z-\sum_{i=1}^{m} y^{i} d x^{i}\right), \quad \xi=2 \frac{\partial}{\partial z}, \\
g=\eta \otimes \eta+1 / 4\left(\sum_{i=1}^{m}\left(d x^{i} \otimes d x^{i}+d y^{i} \otimes d y^{i}\right)\right), \\
\phi_{0}\left(\sum_{i=1}^{m}\left(X_{i} \frac{\partial}{\partial x^{i}}+Y_{i} \frac{\partial}{\partial y^{i}}\right)+Z \frac{\partial}{\partial z}\right)=\sum_{i=1}^{m}\left(Y_{i} \frac{\partial}{\partial x^{i}}-X_{i} \frac{\partial}{\partial y^{i}}\right)+\sum_{i=1}^{m} Y_{i} y^{i} \frac{\partial}{\partial z},
\end{gathered}
$$


where $\left(x^{i}, y^{i}, z\right), i=1 \ldots m$ are the cartesian coordinates. It is well known that

$$
\left\{2 \frac{\partial}{\partial y^{i}}, 2\left(\frac{\partial}{\partial x^{i}}+y^{i} \frac{\partial}{\partial z}\right), \quad \xi\right\}
$$

is an orthonormal basis of $T \mathbf{R}^{2 m+1}$ such that $\phi_{0}\left(2 \frac{\partial}{\partial y^{i}}\right)=2\left(\frac{\partial}{\partial x^{i}}+y^{i} \frac{\partial}{\partial z}\right)$. Such a basis is called a $\phi_{0}$-basis.

When $m$ is even, we define the endomorphism $\phi_{1}$ given by:

$$
\begin{gathered}
\phi_{1}\left(X_{1}, \ldots, X_{m}, Y_{1}, \ldots, Y_{m}, Z\right)=\left(-X_{2}, X_{1}, \ldots,-X_{m}, X_{m-1},\right. \\
\left.Y_{2},-Y_{1}, \ldots, Y_{m},-Y_{m-1}, y^{2} X_{1}-y^{1} X_{2}+\cdots+y^{m} X_{m-1}-y^{m-1} X_{m}\right) .
\end{gathered}
$$

It is easy to show that $\left(\mathbf{R}^{2 m+1}, \phi_{1}, \xi, \eta, g\right)$ is an almost contact metric manifold. Nevertheless, $\left(\mathbf{R}^{2 m+1}, \phi_{1}, \xi, \eta, g\right)$ is not a contact metric manifold. We obtain this as a particular case of the following proposition:

Proposition 3.3. Let $(\tilde{M}, \phi, \xi, \eta, g)$ be a contact metric manifold. If there exists another almost contact metric structure $(\bar{\phi}, \xi, \eta, g)$ on $\widetilde{M}$ such that $(\widetilde{M}, \bar{\phi}, \xi, \eta, g)$ is a contact metric manifold, then $\bar{\phi}=\phi$.

Proof. The result follows easily from the equation $\Phi=\mathrm{d} \eta=\bar{\Phi}$, where $\Phi$ and $\Phi$ denote the fundamental 2 -forms of $(\tilde{M}, \phi, \xi, \eta, g)$ and $(\tilde{M}, \bar{\phi}, \xi, \eta, g)$, respectively.

It can be also proved that $\left(\mathbf{R}^{2 m+1}, \phi_{1}, \xi, \eta, g\right)$ is not normal, since $\left[\phi_{1}, \phi_{1}\right]=$ $2 \mathrm{~d} \eta \otimes \xi$. Moreover, the fundamental 2-form $\Phi_{1}$ is closed and so, we can obtain

$$
g\left(\left(\widetilde{\nabla}_{X} \phi_{1}\right) Y, Z\right)=-2 \eta(X) \mathrm{d} \eta\left(Z, \phi_{1} Y\right)-\eta(Y) \mathrm{d} \eta\left(Z, \phi_{1} X\right)-\eta(Z) \mathrm{d} \eta\left(X, \phi_{1} Y\right),
$$

for any $X, Y, Z \in T \mathbf{R}^{2 m+1}$.

By using the above structures, we find the following method to obtain slant submanifolds, with given slant angle, in a certain almost contact metric manifold.

Example 3.4. For any constant $\theta$, we define $\phi_{0, \theta}$ and $\phi_{1, \theta}$ by

$$
\begin{aligned}
\phi_{0, \theta} & =(\cos \theta) \phi_{0}+(\sin \theta) \phi_{1}, \\
\phi_{1, \theta} & =(\cos \theta) \phi_{1}+(\sin \theta) \phi_{0},
\end{aligned}
$$

respectively. Then, $\left(\phi_{0, \theta}, \xi, \eta, g\right)$ and $\left(\phi_{1, \theta}, \xi, \eta, g\right)$ are two almost contact metric structures on $\mathbf{R}^{2 m+1}$, for any even number $m$. In particular, if $m=2$, then, any invariant submanifold $M$ of dimension 3 in $\left(\mathbf{R}^{5}, \phi_{0}, \xi, \eta, g\right)\left(\operatorname{resp} .\left(\mathbf{R}^{5}, \phi_{1}, \xi, \eta, g\right)\right)$ is a minimal slant submanifold (resp. slant submanifold), with slant angle $\theta$, in $\left(\mathbf{R}^{5}, \phi_{0, \theta}, \xi, \eta, g\right)\left(\operatorname{resp} .\left(\mathbf{R}^{5}, \phi_{1, \theta}, \xi, \eta, g\right)\right)$.

To obtain examples in a Sasakian manifold, we can prove, by a direct calculation, the following theorem. 
Theorem 3.5. Suppose that

$$
x(u, v)=\left(f_{1}(u, v), f_{2}(u, v), f_{3}(u, v), f_{4}(u, v)\right)
$$

defines a slant surface $S$ in $\mathbf{C}^{2}$ with its usual Kaehlerian structure, such that $\partial / \partial u$ and $\partial / \partial v$ are non-zero and perpendicular. Then,

$$
y(u, v, t)=2\left(f_{1}(u, v), f_{2}(u, v), f_{3}(u, v), f_{4}(u, v), t\right)
$$

defines a three-dimensional slant submanifold $M$ in $\left(\mathbf{R}^{5}, \phi_{0}, \xi, \eta, g\right)$, such that, if we put

$$
e_{1}=\frac{\partial}{\partial u}+\left(2 f_{3} \frac{\partial f_{1}}{\partial u}+2 f_{4} \frac{\partial f_{2}}{\partial u}\right) \frac{\partial}{\partial t}
$$

and

$$
e_{2}=\frac{\partial}{\partial v}+\left(2 f_{3} \frac{\partial f_{1}}{\partial v}+2 f_{4} \frac{\partial f_{2}}{\partial v}\right) \frac{\partial}{\partial t}
$$

then $\left\{e_{1}, e_{2}, \xi\right\}$ is an orthogonal basis of the tangent bundle of the submanifold.

REMARK 3.6. Note that if $S$ is not totally real, then, the distribution spanned by $e_{1}$ and $e_{2}$ is not integrable and so, $M$ cannot be the Riemannian product of $S$ by $\mathbf{R}$. This fact is coherent with Proposition 3.2.

Then, from Theorem 3.5 and Examples 2.1, 2.3, 2.4 and 2.5 of [2], we obtain the following examples in $\mathbf{R}^{5}$ with its usual Sasakian structure:

ExAmple 3.7. For any $\theta \in[0, \pi / 2]$,

$$
x(u, v, t)=2(u \cos \theta, u \sin \theta, v, 0, t)
$$

defines a minimal three-dimensional slant submanifold with slant angle $\theta$ and with scalar curvature $\tau=-\left(\cos ^{2} \theta\right) / 3$.

EXAMPLE 3.8. For any constant $k$,

$$
x(u, v, t)=2\left(e^{k u} \cos u \cos v, e^{k u} \sin u \cos v, e^{k u} \cos u \sin v, e^{k u} \sin u \sin v, t\right)
$$

defines a slant submanifold of dimension 3 with slant angle $\theta=\cos ^{-1}\left(|k| / \sqrt{1+k^{2}}\right)$, scalar curvature $\tau=-k^{2} /\left(3\left(1+k^{2}\right)\right)$ and non-constant mean curvature given by $|H|=2 e^{-k u} /\left(3 \sqrt{1+k^{2}}\right)$. Hence, the submanifold is not minimal.

EXAmPLE 3.9. For any constant $k$,

$$
x(u, v, t)=2(u, k \cos v, v, k \sin v, t)
$$

defines a slant submanifold $M$ with slant angle $\cos ^{-1}\left(1 / \sqrt{1+k^{2}}\right)$, constant mean curvature $|H|=|k| /\left(3\left(1+k^{2}\right)\right)$ and scalar curvature $\tau=-1 /\left(3\left(1+k^{2}\right)\right)$. Moreover, the following statements are equivalent: (a) $k=0$, (b) $M$ is invariant, (c) $M$ is minimal and (d) $M$ has parallel mean curvature vector. 
EXAmple 3.10. Let $k$ be any positive number and $(g(s), h(s))$ a unit speed plane curve. Then,

$$
x(u, s, t)=2(-k s \sin u, g(s), k s \cos u, h(s), t)
$$

defines a non-minimal proper slant submanifold with slant angle $k / \sqrt{1+k^{2}}$.

In a similar way, we also can obtain examples of slant submanifolds with higher dimension.

EXAMPLE 3.11. For any $k>0$,

$$
x(u, v, w, s, t)=2(u, v, k \sin w, k \sin s, k w, k s, k \cos w, k \cos s, t)
$$

defines a slant submanifold with slant angle $\pi / 4$ in $\left(\mathbf{R}^{9}, \phi_{0}, \xi, \eta, g\right)$.

Example 3.12. For any $\theta \in[0, \pi / 2]$,

$$
x(u, v, w, s, t)=2(u, 0, w, 0, v \cos \theta, v \sin \theta, s \cos \theta, s \sin \theta, t)
$$

defines a 5-dimensional minimal slant submanifold in $\left(\mathbf{R}^{9}, \phi_{0}, \xi, \eta, g\right)$, with slant angle $\theta$.

Moreover, we have the following examples of three-dimensional slant submanifolds in $\left(\mathbf{R}^{7}, \phi_{0}, \xi, \eta, g\right)$ :

ExAmple 3.13. For any $\theta \in[0, \pi / 2]$,

$$
x(u, v, t)=2(u, 0,0, v \cos \theta, v \sin \theta, 0, t)
$$

defines a 3-dimensional minimal slant submanifold, with slant angle $\theta$, in $\mathbf{R}^{7}$ with its usual Sasakian structure.

By proceeding as in Theorem 3.5 we find examples of slant submanifolds in the almost contact metric manifold $\left(\mathbf{R}^{5}, \phi_{1}, \xi, \eta, g\right)$.

EXAmple 3.14. For any nonzero real numbers $p$ and $q$, we consider the following immersion from $\mathbf{R} \times(0, \infty) \times \mathbf{R}$ into $\mathbf{R}^{5}$ defined by:

$$
x(u, v, t)=2(p v \sin u, p v \cos u, v \sin q u, v \cos q u, t) .
$$

Then, the immersion $x$ gives a slant three-dimensional submanifold in the almost contact metric manifold $\left(\mathbf{R}^{5}, \phi_{1}, \xi, \eta, g\right)$.

EXAmple 3.15. For any $\theta \in[0, \pi / 2]$,

$$
x(u, v, t)=2(u \cos \theta, v, u \sin \theta, 0, t)
$$

defines a minimal slant submanifold with slant angle $\theta$ in $\left(\mathbf{R}^{5}, \phi_{1}, \xi, \eta, g\right)$. 
Example 3.16. For any $\theta \in[0, \pi / 2]$,

$$
x(u, v, t)=2(u \cos \theta, v, 0, u \sin \theta, t)
$$

defines a minimal slant submanifold with slant angle $\theta$ in $\left(\mathbf{R}^{5}, \phi_{1}, \xi, \eta, g\right)$.

With respect to Example 3.16, it can be proved that, for any $\theta \in[0, \pi / 2]$, we also obtain a slant submanifold in $\left(\mathbf{R}^{5}, \phi_{0}, \xi, \eta, g\right)$, with slant angle $\pi / 2-\theta$. Hence, if $\theta=\pi / 4$, then we have slant immersions in two different almost contact metric manifolds, with the same slant angle.

Finally, we show another example of slant submanifold in $\mathbf{R}^{5}$ with its usual Sasakian structure, which is not given by the method of Theorem 3.5.

EXAmple 3.17. For any $\theta \in[0, \pi / 2]$,

$$
x(u, v, t)=2(u, 0, v \cos \theta, v \sin \theta, 2 u v \cos \theta+t)
$$

defines a slant submanifold in $\mathbf{R}^{5}$ with slant angle $\theta$.

4. Slant submanifolds of $\boldsymbol{K}$-contact manifolds. In this section, we will study the value of $\nabla Q$ for slant submanifolds of a $K$-contact manifold, in order to obtain a characterization theorem for such submanifolds.

In the complex case, slant submanifolds satisfy the expression $\nabla Q=0$ (see [2]). Now, in the $K$-contact case, the situation is quite different.

Proposition 4.1. Let $M$ be a slant submanifold of a K-contact manifold $\tilde{M}$. Then, $\nabla Q=0$ if and only if $M$ is an anti-invariant submanifold.

Proof. Denote by $\theta$ the slant angle of $M$. Given $X, Y \in T M,(2.1)$ implies:

$$
Q \nabla_{X} Y=-\cos ^{2} \theta \nabla_{X} Y+\cos ^{2} \theta \eta\left(\nabla_{X} Y\right) \xi
$$

On the other hand, by taking the covariant derivative of (2.1), we have

$$
\begin{gathered}
\nabla_{X} Q Y=-\cos ^{2} \theta \nabla_{X} Y+\cos ^{2} \theta \eta\left(\nabla_{X} Y\right) \xi+ \\
+\cos ^{2} \theta g\left(Y, \nabla_{X} \xi\right) \xi+\cos ^{2} \theta \eta(Y) \nabla_{X} \xi
\end{gathered}
$$

since $X(\eta(Y))=\eta\left(\nabla_{X} Y\right)+g\left(Y, \nabla_{X} \xi\right)$.

Hence, $\nabla Q=0$ if and only if (4.1) equals (4.2), which is equivalent to $\nabla_{X} \xi=0$ for any $X \in T M$. But, since $\widetilde{M}$ is a $K$-contact manifold, we have $\nabla_{X} \xi=-T X$ and so, the result holds.

In fact, by using (4.1), (4.2) and $\nabla_{X} \xi=-T X$, we can see that, if $M$ is a slant submanifold of a $K$-contact manifold $\widetilde{M}$, then

$$
\left(\nabla_{X} Q\right) Y=\cos ^{2} \theta(g(X, T Y) \xi-\eta(Y) T X) .
$$


for any $X, Y \in T M$, where $\theta$ denotes the slant angle of $M$. This expression will be useful to characterize slant submanifolds.

The following lemma has been proved in [7]:

LEMMA 4.2. Let $M$ be a slant submanifold of an almost contact metric manifold $\tilde{M}$. Denote by $\theta$ the slant angle of $M$. Then, at each point x of $M,\left.Q\right|_{\mathcal{D}}$ has only one eigenvalue $\lambda_{1}=-\cos ^{2} \theta$.

THEOREM 4.3. Let $M$ be a submanifold of a K-contact manifold $\tilde{M}$ such that $\xi \in T M$. Then, $M$ is slant if and only if

1. The endomorphism $\left.Q\right|_{\mathcal{D}}$ has only one eigenvalue at each point of $M$.

2. There exists a function $\lambda: M \rightarrow[0,1]$ such that

$$
\left(\nabla_{X} Q\right) Y=\lambda(g(X, T Y) \xi-\eta(Y) T X)
$$

for any $X, Y \in T M$.

Moreover, in this case, if $\theta$ is the slant angle of $M$, we have $\lambda=\cos ^{2} \theta$.

Proof. Statements 1 and 2 follow directly from Lemma 4.2 and (4.3), respectively. Conversely, let $\lambda_{1}(x)$ be the only eigenvalue of $\left.Q\right|_{\mathcal{D}}$ at each point $x \in M$. Let $Y \in \mathcal{D}$ be an unit eigenvector associated with $\lambda_{1}$, i.e., $Q Y=\lambda_{1} Y$. Then, by virtue of statement 2, we have

$$
X\left(\lambda_{1}\right) Y+\lambda_{1} \nabla_{X} Y=\nabla_{X}(Q Y)=Q\left(\nabla_{X} Y\right)+\lambda g(X, T Y) \xi
$$

for any $X \in T M$, since $Y \in \mathcal{D}$. Since both $\nabla_{X} Y$ and $Q\left(\nabla_{X} Y\right)$ are perpendicular to $Y$, we conclude that $\lambda_{1}$ is constant on $M$.

By virtue of (2.1), to prove that $M$ is slant it is enough to show that there is a constant $\mu$ such that $Q=-\mu I+\mu \eta \otimes \xi$. Let $X$ be in $T M$. Then, $X=\tilde{X}+\eta(X) \xi$, where $\widetilde{X}=X-\eta\left(\underset{\tilde{X}}{)} \xi \in \mathcal{D}\right.$. Hence, $Q X=Q \widetilde{X}$. Since $\left.Q\right|_{\mathcal{D}}=\lambda_{1} I$, we have $Q \widetilde{X}=\lambda_{1} \widetilde{\widetilde{X}}$ and so $Q X=\lambda_{1} \widetilde{X}=\lambda_{1} X-\lambda_{1} \eta(X) \xi$. By taking $\mu=-\lambda_{1}$, we obtain the result. Moreover, if $M$ is slant, by virtue of (4.3), it must be $\lambda=-\lambda_{1}=\mu=\cos ^{2} \theta$, where $\theta$ denotes the slant angle of $M$.

COROLlary 4.4. Let $M$ be a submanifold of dimension 3 of a K-contact manifold $\tilde{M}$ such that $\xi \in T M$. Then, $M$ is slant if and only if there exists a function $\lambda: M \rightarrow[0,1]$ such that

$$
\left(\nabla_{X} Q\right) Y=\lambda(g(X, T Y) \xi-\eta(Y) T X)
$$

for any $X, Y \in T M$. Moreover, in this case, if $\theta$ is the slant angle of $M$, we have $\lambda=\cos ^{2} \theta$.

Proof. It follows directly from Theorem 4.3 since it iseasy to prove that, if $\operatorname{dim} M=3$, then $\left.Q\right|_{\mathcal{D}}$ has only one eigenvalue at each point of $M$. 
5. Slant submanifolds with dimension 3 . Three-dimensional slant submanifolds make up the first non-trivial class of slant submanifolds in almost contact metric manifolds. Now, we are going to characterize these submanifolds by giving the values of $\nabla T$ and $\nabla N$.

THEOREM 5.1. Let $M$ be a submanifold of dimension 3 in a K-contact manifold $\widetilde{M}$ such that $\xi \in T M$. Then, the following three statements are equivalent:

1. $M$ is neither an invariant nor an anti-invariant submanifold and there exists a function $\lambda: M \rightarrow[0,1]$ such that

$$
\left(\nabla_{X} T\right) Y=\lambda(g(X, Y) \xi-\eta(Y) X)
$$

for any $X, Y \in T M$.

2. $M$ is a proper slant submanifold such that

$$
\left(\nabla_{X} T\right) Y=\cos ^{2} \theta(g(X, Y) \xi-\eta(Y) X)
$$

for any $X, Y \in T M$, where $\theta$ denotes the slant angle.

3. $M$ is proper slant.

Proof. By virtue of Corollary 4.4 and the relation between (1.7) and (1.8), it follows that statements 1 and 2 are equivalent. It is obvious that 2 implies 3 . The converse has been proved by A. Lotta (see Theorem 2.2 of [6]).

REMARK 5.2. The conditions of Theorem 5.1 are sufficient but not necessary. In fact, we can prove that 5-dimensional slant submanifolds given by Examples 3.11 and 3.12 satisfy (5.1).

Similarly, we can see that it is not necessary for $\tilde{M}$ to have a $K$-contact structure. If $\theta=\pi / 4$, Example 3.16 defines a $\pi / 4$-slant submanifold in $\left(\mathbf{R}^{5}, \phi_{1}, \xi, \eta, g\right)$ such that it satisfies (5.1).

However, Theorem 5.1 does not hold in any almost contact metric manifold. To show this, it is enough to see that submanifolds given by Example 3.15 satisfy $\nabla T=0$.

Note that the proof of Theorem 5.1 also works when $M$ is an invariant submanifold, since it is enough to have $\cos \theta \neq 0$. In the anti-invariant case, $T \equiv 0$ and so, $\left(\nabla_{X} T\right) Y=0=\cos ^{2} \theta(g(X, Y) \xi-\eta(Y) X)$, for any $X, Y \in T M$, since $\theta=\pi / 2$. Moreover, in this case, we do not need for $M$ to have dimension 3.

Combining this fact with Theorem 5.1 and the equivalence between (1.9) and (1.10), we obtain the following characterization of slant submanifolds with dimension 3 in terms of the Weingarten map.

COROllary 5.3. Let $M$ be a 3-dimensional submanifold in a Sasakian manifold $\widetilde{M}$ such that $\xi \in T M$. Then, $M$ is slant if and only if it exists a function $C: M \rightarrow[0,1]$ such that

$$
A_{N Y} X=A_{N X} Y+C \widetilde{R}(X, Y) \xi
$$

for any $X, Y \in T M$. Moreover, in this case, if $\theta$ is the slant angle of $M$, then we have $C=\sin ^{2} \theta$. 
If $M$ is an invariant submanifold of a Sasakian manifold, then (5.1) holds with $\theta=0$ and $\nabla N=0$ automatically. On the other hand, if $M$ is an anti-invariant submanifold, it is obvious that $\nabla T=0$, i.e., (5.1) holds with $\theta=\pi / 2$. We also know that $\left(\nabla_{X} N\right) Y=n \sigma(X, Y)$ for any $X, Y \in T M$. The following lemma show us sufficient conditions to obtain $\nabla N=0$.

Lemma 5.4. Let $M$ be an anti-invariant submanifold of a Sasakian manifold $\tilde{M}$. Suppose that $\operatorname{dim} M=3, \operatorname{dim} \widetilde{M}=5$ and $\xi \in T M$. Then, $\nabla N=0$.

Proof. By virtue of (2.4), if we choose a local orthonormal frame $\left\{e_{1}, e_{2}, \xi\right\}$ of $T M$, then we have that $\left\{N e_{1}, N e_{2}\right\}$ is a local orthonormal frame of $T^{\perp} M$ and so, $n=0$. Consequently, we obtain $\nabla N=0$.

Now, we are going to study the value of $\nabla N$ for a three-dimensional slant submanifold $M$ of a Sasakian manifold $\widetilde{M}$ with $\operatorname{dim} \widetilde{M}=5$.

Suppose that $M$ is proper slant with slant angle $\theta$. Then, for a unit tangent vector field $e_{1}$ of $M$, perpendicular to $\xi$, we put

$$
e_{2}=(\sec \theta) T e_{1}, \quad e_{3}=\xi, \quad e_{4}=(\csc \theta) N e_{1} \quad \text { and } \quad e_{5}=(\csc \theta) N e_{2} .
$$

Then, $e_{1}=-(\sec \theta) T e_{2}$ and, by virtue of (2.3) and (2.4), $e_{1}, e_{2}, e_{3}, e_{4}, e_{5}$ form an orthonormal frame such that $e_{1}, e_{2}, e_{3}$ are tangent to $M$ and $e_{4}, e_{5}$ are normal to $M$. We call such an orthonormal frame an adapted slant frame. We also have:

$$
t e_{4}=-\sin \theta e_{1}, \quad t e_{5}=-\sin \theta e_{2}, \quad n e_{4}=-\cos \theta e_{5}, \quad n e_{5}=\cos \theta e_{4} .
$$

If we put $\sigma_{i j}^{r}=g\left(\sigma\left(e_{i}, e_{j}\right), e_{r}\right), i, j=1,2,3, r=4,5$, then we have the following lemma:

LEMMA 5.5. In the above conditions, we have:

$$
\begin{array}{ll}
\sigma_{12}^{4}=\sigma_{11}^{5}, & \sigma_{22}^{4}=\sigma_{12}^{5}, \\
\sigma_{13}^{4}=\sigma_{23}^{5}=-\operatorname{sen} \theta, & \sigma_{23}^{4}=\sigma_{13}^{5}=\sigma_{33}^{4}=\sigma_{33}^{5}=0 .
\end{array}
$$

Proof. We obtain (5.2) by virtue of Corollary 5.3 while (5.3) holds because $\tilde{M}$ is a $K$-contact manifold.

THEOREM 5.6. Let $M$ be a submanifold of a Sasakian manifold $\tilde{M}$ such that $\xi \in T M, \operatorname{dim} M=3$ and $\operatorname{dim} \widetilde{M}=5$.

1. If $M$ is a minimal proper slant submanifold of $\tilde{M}$, then

$$
\left(\nabla_{X} N\right) Y=2 \eta(X) N T Y+\eta(Y) N T X,
$$

for any $X, Y \in T M$.

2. Conversely, suppose that there is an eigenvalue $\lambda$ of $\left.Q\right|_{\mathcal{D}}$ at each point of $M$ such that $\lambda \in(-1,0)$. In this case, if (5.4) holds then $M$ is a minimal proper slant submanifold of $\tilde{M}$. 
Proof. To prove statement 1 , we choose $e_{1}, \ldots, e_{5}$ an adapted slant frame. Then, from (1.10), (5.2), (5.3) and a direct computation we may obtain (5.4).

To see statement 2 , we choose an unit local vector field $e_{1}$, perpendicular to $\xi$, such that

$$
T^{2} e_{1}=-\cos ^{2} \theta_{1} e_{1}
$$

where $\theta_{1}=\theta\left(e_{1}\right) \in(0, \pi / 2)$ denotes the Wirtinger angle of $e_{1}$. Define $e_{1}, \ldots, e_{5}$ by:

$$
e_{2}=\left(\sec \theta_{1}\right) T e_{1}, \quad e_{3}=\xi, \quad e_{4}=\left(\csc \theta_{1}\right) N e_{1} \quad \text { and } \quad e_{5}=\left(\csc \theta_{1}\right) N e_{2}
$$

It is easy to prove that $e_{1}, \ldots, e_{5}$ is a local orthonormal frame and:

$$
t e_{4}=-\sin \theta_{1} e_{1}, \quad n e_{4}=-\cos \theta_{1} e_{5}, \quad t e_{5}=-\sin \theta_{1} e_{2} \quad y \quad n e_{5}=\cos \theta_{1} e_{4}
$$

Since $\left(\nabla_{X} N\right) Y=2 \eta(X) N T Y+\eta(Y) N T X$, for any $X, Y \in T M$, we know that

$$
A_{V} T Y=-A_{n V} Y+2 g(Y, \operatorname{tn} V) \xi+\eta(Y) \operatorname{tn} V,
$$

for any $Y \in T M$ and any $V \in T M^{\perp}$. Therefore, we find:

$$
A_{N e_{1}} e_{2}=\sec \theta_{1} \sin \theta_{1} A_{e_{4}} T e_{1}=\tan \theta_{1} A_{e_{4}} T e_{1}=\sin \theta_{1} A_{e_{5}} e_{1}=A_{N e_{2}} e_{1} .
$$

Moreover, we have $A_{N e_{1}} e_{3}=\sin \theta_{1} A_{e_{4}} e_{3}=-\sin ^{2} \theta_{1} e_{1}$ and $A_{N e_{2}} e_{3}=-\operatorname{sen}^{2} \theta_{1} e_{2}$.

Hence, by a direct computation, we can prove that

$$
A_{N Y} X=A_{N X} Y-\sin ^{2} \theta_{1}(\eta(X) Y-\eta(Y) X)
$$

for any $X, Y \in T M$ and so, by applying Corollary 5.3, we know that $M$ is proper slant, with slant angle $\theta_{1}$. It is easy to show that $M$ is also minimal.

Note that (5.4) holds directly in the invariant and anti-invariant cases, since $\nabla N=0$.

REMARK 5.7. The dimension conditions of Theorem 5.6 are sufficient but not necessary. To show this, it is enough to see that every slant submanifold given by Examples 3.13 and 3.12 satisfies (5.4).

Similarly, it is not necessary for $\tilde{M}$ to have a Sasakian structure. If $\theta=\pi / 4$, Example 3.16 defines a minimal $\pi / 4$-slant submanifold $M$ in $\left(\mathbf{R}^{5}, \phi_{1}, \xi, \eta, g\right)$, such that $M$ satisfies (5.4).

However, Theorem 5.6 does not hold in any almost contact metric manifold, as we can see in Example 3.15. In this example, we find three-dimensional minimal proper slant submanifolds in $\left(\mathbf{R}^{5}, \phi_{1}, \xi, \eta, g\right)$ such that $\nabla N=0$. 


\section{REFERENCES}

1. D. E. Blair, Contact manifolds in Riemannian geometry, Lecture Notes in Mathematics No. 509 (Springer-Verlag, 1976).

2. B. Y. Chen, Geometry of slant submanifolds (Katholieke Universiteit Leuven, 1990).

3. B. Y. Chen, Slant immersions, Bull. Austral. Math. Soc. 41 (1990), 135-147.

4. B. Y. Chen and Y. Tazawa, Slant surfaces with codimension 2, Ann. Fac. Sci. Toulouse Math. XI(3) (1990), 29-43.

5. B. Y. Chen and Y. Tazawa, Slant submanifolds in complex euclidean spaces, Tokyo J. Math. 14(1) (1991), 101-120.

6. A. Lotta, Three-dimensional slant submanifolds of K-contact manifolds, Balkan J. Geom. Appl.

7. A. Lotta, Slant submanifolds in contact geometry, Bull. Math. Soc. Roumanie 39 (1996), 183-198.

8. A. Lotta and A. M. Pastore, Foliations of the Sasakian space $\mathbf{R}^{2 n+1}$ by minimal slant submanifolds, Preprint.

9. S. Maeda, Y. Ohnita and S. Udagawa, On slant immersions into Kähler manifolds, Kodai Math. J. 16 (1993), 205-219.

10. K. Yano and M. Kon, Anti-invariants submanifolds, Lecture Notes in Pure and Applied Mathematics Vol. 21 (Marcel Dekker, 1976). 